\title{
Radiosurgery for melanoma brain metastases in the ipilimumab era and the possibility of longer survival
}

\author{
Clinical article
}

\author{
Jonathan P. S. Knisely, M.D., ${ }^{1}$ James B. Yu, M.D., ${ }^{3,4}$ Jaclyn Flanigan, M.D., ${ }^{2,3}$ \\ Mario Sznol, M.D., ${ }^{2,3}$ Harriet M. Kluger, M.D., ${ }^{2,3}$ and Veronica L. S. Chiang, M.D. ${ }^{3,5}$ \\ ${ }^{1}$ Department of Radiation Medicine, Hofstra University North Shore-LIJ School of Medicine, Hofstra \\ University, Manhasset, New York; ${ }^{2}$ Department of Internal Medicine, Section of Medical Oncology; \\ Departments of ${ }^{4}$ Therapeutic Radiology and ${ }^{5}$ Neurosurgery, Yale University School of Medicine; \\ and ${ }^{3}$ Yale Cancer Center, New Haven, Connecticut
}

Object. A prospectively collected cohort of 77 patients who underwent definitive radiosurgery between 2002 and 2010 for melanoma brain metastases was retrospectively reviewed to assess the impact of ipilimumab use and other clinical variables on survival.

Methods. The authors conducted an institutional review board-approved chart review to assess patient age at the time of brain metastasis diagnosis, sex, primary disease location, initial radiosurgery date, number of metastases treated, performance status, systemic therapy and ipilimumab history, whole-brain radiation therapy (WBRT) use, follow-up duration, and survival at the last follow-up. The Diagnosis-Specific Graded Prognostic Assessment (DSGPA) score was calculated for each patient based on performance status and the number of brain metastases treated.

Results. Thirty-five percent of the patients received ipilimumab. The median survival in this group was 21.3 months, as compared with 4.9 months in patients who did not receive ipilimumab. The 2-year survival rate was $47.2 \%$ in the ipilimumab group compared with $19.7 \%$ in the nonipilimumab group. The DS-GPA score was the most significant predictor of overall survival, and ipilimumab therapy was also independently associated with an improvement in the hazard for death $(\mathrm{p}=0.03)$.

Conclusions. The survival of patients with melanoma brain metastases managed with ipilimumab and definitive radiosurgery can exceed the commonly anticipated 4-6 months. Using ipilimumab in a supportive treatment paradigm of radiosurgery for brain oligometastases was associated with an increased median survival from 4.9 to 21.3 months, with a 2 -year survival rate of $19.7 \%$ versus $47.2 \%$. This association between ipilimumab and prolonged survival remains significant even after adjustment for performance status without an increased need for salvage WBRT. (http://thejns.org/doi/abs/10.3171/2012.5.JNS111929)

\section{KEY WoRdS • melanoma • brain metastasis • radiosurgery • Gamma Knife • ipilimumab • oncology}

$\mathrm{M}$ ELANOMA, a deadly and aggressive cancer, is the third most common cause of brain metastases. The relative incidence of melanoma brain metastases is between $10 \%$ and $40 \%$ in patients with Stage IV disease, and over $75 \%$ with Stage IV melanoma have brain metastases at autopsy. ${ }^{8,10,14}$ Historically, not only have melanoma brain metastases had a poor prognosis given the difficulty in controlling systemic disease, but the brain metastases have also contributed directly to the cause of death in between $20 \%$ and $54 \%$ of patients with metastatic melanoma. ${ }^{12}$

Recently, 2 large randomized studies showed that ipilimumab, a fully human antibody that binds to CTLA-4, improves survival in patients with unresectable Stage III

Abbreviations used in this paper: CTLA-4 = cytotoxic T lymphocyte-associated antigen 4; DS-GPA = Diagnosis-Specific Graded Prognostic Assessment; ECOG PS = Eastern Cooperative Oncology Group performance status; GKS = Gamma Knife surgery; KPS = Karnofsky Performance Scale; SRS = stereotactic radiosurgery; $\mathrm{WBRT}=$ whole-brain radiation therapy. and Stage IV melanoma. ${ }^{5,9}$ One was the first randomized clinical trial to demonstrate an overall survival benefit for any systemic therapy in patients with metastatic melanoma. Survival was improved from 6.4 to $\geq 10$ months for patients $(p \leq 0.003)$ in the 2 arms that were treated with ipilimumab. A significant proportion $(22.0 \%)$ of those who received ipilimumab survived more than 2 years, as compared with $13.7 \%$ of those who did not. Only $12.1 \%$ of the patients had brain metastases at the time of study entry, and the authors did not specifically report on brain metastasis management or outcomes for this subset. In a second reported study, patients with previously untreated, unresectable Stage III or Stage IV melanoma were randomized to receive dacarbazine with or without ipilimumab. ${ }^{9}$ Overall survival was significantly longer in the group receiving ipilimumab (11.2 vs 9.1 months), but patients with brain metastases were excluded from this trial. These important studies, which documented that the use of novel molecular therapies for patients with metastatic melanoma can improve survival, leave unanswered the question of the efficacy of these interventions in patients 
with locally treated or untreated melanoma brain metastases.

The conventional management of melanoma brain metastases has included resection when feasible and WBRT especially when multiple lesions are seen. Cellsurvival curves generated after exposing melanoma cells to doses of ionizing radiation commonly used in WBRT have shown that these cells are often able to repair damage from small radiation doses, making them less radiosensitive than other common brain metastasis histologies. ${ }^{3}$ Therefore, SRS, which treats only the visible brain metastases but with a single higher dose of radiation, appears to be better suited for addressing melanoma brain metastases. At our center we have moved toward an approach that utilizes Gamma Knife SRS for the treatment of multiple brain metastases, as long as the patient's performance status is good, and the use of WBRT as a salvage measure only for rapidly progressive CNS failure.

These data beg several questions. First, is the systemic T-cell effect that ipilimumab unleashes able to translate into survival increases, and does ipilimumab use confer a survival advantage in melanoma patients with brain metastases? Second, for patients who have prolonged survival due to a response to ipilimumab, what is the course of subsequent brain metastases? At the Yale Cancer Center, the use of ipilimumab in a number of clinical trials was initiated in 2004. To address our questions we reviewed our experience with the use of SRS in patients with melanoma brain metastases who did or did not receive ipilimumab.

\section{Methods}

Medical records for patients treated with GKS between November 2002 and November 2010 for melanoma brain metastases at Yale-New Haven Hospital were reviewed after obtaining approval from the Yale Human Investigations Committee. We identified 77 patients with melanoma brain metastases treated with SRS. Serum lactate dehydrogenase levels or the extent of systemic metastatic disease were not taken into account in evaluating patients for possible SRS. All visualized brain metastases, regardless of the number in each patient, were treated with single-fraction GKS plans. During follow-up, when new metastatic deposits were visualized on MRI studies of the brain, a decision was made about salvage management (resection, repeat SRS, and WBRT) based on the patient's other clinical parameters. Repeat SRS was the treatment modality of choice for new metastases as long as the patient's performance status remained good.

Information on systemic therapy was collected. Patients were treated with a number of immune therapies, chemotherapies, and biological therapies, including sorafenib (given with carboplatin and paclitaxel), dasatinib, and vemurafenib. Clinical trial use of ipilimumab alone or with a peptide vaccine began in 2004 and continued through the end of this study. Most patients were treated in compassionate use trials. Those who received ipilimumab in the compassionate use studies were required to have an ECOG PS $\leq 2$ and measurable systemic disease according to Response Evaluation Criteria in Solid Tumors (RECIST).
The newly reported DS-GPA ${ }^{13}$ for melanoma was used as a pretreatment predictor of outcome (Table 1) and is calculated by assigning point values of 0,1 , or 2 to three classes of two variables: KPS score and number of brain metastases. A KPS score of 90-100 (ECOG PS of 0 ) is assigned a value of 2 points, whereas a KPS score of $70-80$ is assigned a value of 1 point. A KPS score below 70 (ECOG PS $\geq 3$ ) is assigned a value of 0 points. The other component of the DS-GPA assigns points based on the number of brain metastases. A single brain metastasis is assigned a value of 2,2-3 metastases a value of 1 , and $>3$ metastases a value of 0 . Adding the points from these two components gives a DS-GPA score between 0 and 4 . This assessment has been found to supersede prior scales in the prediction of survival outcome.

Survival from the date of the first radiosurgical treatment was assessed in relation to the use of ipilimumab, patient age and sex, craniotomy status, the use of WBRT, ECOG PS, the number of brain metastases at the first radiosurgical intervention, and the DS-GPA score for melanoma, which was calculated for all patients. ${ }^{13}$

Univariate Cox proportional hazards analyses for survival were performed for the entire follow-up period, while also using a 2-year cutoff. This cutoff was used so that a real and temporary improvement in overall survival would not be overwhelmed by the ultimate fact that patients with metastatic disease eventually succumb to their disease regardless of treatment. These parallel analyses showed that a low ECOG PS, a high DS-GPA score, and the use of ipilimumab were associated with improved survival. Because the DS-GPA incorporates a patient's performance status and because the multivariate Cox proportional hazards model for survival that incorporated DS-GPA had a likelihood ratio chi-square value of 32.44 (vs 25.19 for the ECOG PS-containing model), we used the DS-GPA rather than the ECOG PS for subsequent analytical modeling.

A secondary analysis was performed to look at CNS failure rates requiring retreatment subsequent to the first GK treatment. Overall survival was calculated using the Kaplan-Meier method. The use of SRS and WBRT was recorded, and death dates were known for all patients. Relationships of covariates to overall survival were analyzed using Cox proportional hazards analysis. The proportional hazards assumption was confirmed for univariate and multivariate models. All covariates with a $p<0.10$ were included in the multivariate model. All statistical analysis was performed using STATA/SE 9.2 (StataCorp).

\section{Results}

Patient characteristics are outlined in Table 2. Almost two-thirds were male, and the median age at brain metastasis diagnosis was 61 years. Twenty-seven patients $(35 \%)$ received ipilimumab, and these patients tended to be younger ( $\mathrm{p}=0.047,2$-sided $\mathrm{t}$-test) than those who did not receive the drug. There was no significant difference in DS-GPA scores between the 2 groups ( $p=0.21$, chisquare test).

The median survival for the entire cohort was 8.8 months (95\% CI 4.93-18.4). For patients who received 
Melanoma, stereotactic radiosurgery, and ipilimumab

TABLE 1: Diagnosis-Specific Graded Prognostic Assessment scoring system for melanoma

\begin{tabular}{lccc}
\hline \multicolumn{1}{c}{ Variable } & 0 Points & 1 Point & 2 Points \\
\hline KPS score & $<70$ & $70-80$ & $90-100$ \\
no. of brain metastases & $>3$ & $2-3$ & 1 \\
\hline
\end{tabular}

ipilimumab, median survival was 21.3 months $(95 \%$ CI 6.43-26.7) compared with 4.9 months (95\% CI 3.3-10.4) for those who did not. Further, 2-year survival was $47.2 \%$ (95\% CI 24.8\%-66.8\%) in the ipilimumab group compared with $19.7 \%$ (95\% CI 9.0\%-33.5\%) in the nonipilimumab group. Kaplan-Meier survival curves are shown in Fig. 1.

Of the 27 patients who received ipilimumab, 11 were treated with the drug before the SRS treatment and 16 patients started the drug after SRS. A Cox proportional hazards analysis of the subset that received ipilimumab revealed no difference in the hazard for death whether the drug was started before or after the first SRS treatment. The median survival for patients who received ipilimumab before SRS was 19.8 months (95\% CI 1.5 months-not yet reached upper limit), and for those who received ipilimumab after SRS the median survival was 21.3 months (95\% CI 15.7 months-not yet reached upper limit). There were no differences in survival according to log-rank analysis $(\mathrm{p}=0.58)$.

Multivariate analyses using Cox proportional hazards modeling are set forth in Table 3. In the overall analysis, the receipt of ipilimumab was not associated with a statistically significant improvement in the hazard for death (HR 0.61, 95\% CI 0.33-1.10, p =0.102), whereas DS-GPA scores of 2, 3, and 4 ( $p<0.001$ for each) were associated with a reduced hazard for death as compared with a DS-GPA score of 0 or 1 . However, the multivariate Cox proportional hazards analysis with data censored at 24 months showed that ipilimumab therapy was independently associated with an improvement in the hazard for death (HR 0.48, 95\% CI 0.24-0.93, $\mathrm{p}=0.03$ ). This was true for the overall cohort that included patients with a better prognosis (DS-GPA scores of 3-4) as well as for patients with worse prognoses as indicated by DS-GPA scores of $0-2$. The median survival among patients with DS-GPA scores of 3-4 was 25.2 months in those who received ipilimumab versus 14.67 months in those who did not. Among patients with DS-GPA scores of 0-2, median survival was 15.67 months among those who took ipilimumab versus 3.03 months in those who did not (Table 4).

When included in the multivariate model, the year of GKS treatment (as a reflection of changing treatment protocols over time) did not change the hazard ratios for ipilimumab use or ECOG PS significantly, as they remained statistically significant. Patient age and sex as well as the use of craniotomy and/or WBRT did not impact survival.

Whole-brain radiation therapy was used in 25 $(32.5 \%)$ of 77 patients. In the cohort that received ipilimumab, $11(40.7 \%)$ of the 27 received WBRT. In the cohort that did not receive ipilimumab, 14 (28.0\%) of the 50 received WBRT. In both groups, about $20 \%$ of patients needed salvage WBRT (6 of 27 for the ipilimumab group vs 8 of 50 for the nonipilimumab group). Ten (37\%) of 27 patients never needed salvage treatment with SRS, surgery, or WBRT. The median number of subsequent SRS treatments per patient was 2 (range 1-5). The median interval between the first and subsequent SRS treatments was 6 months (range 2-24 months). A median of 2 brain metastases (range 1-16) required treatment at the first SRS session; in subsequent SRS sessions, a median of 8 metastases (range 1-52) needed salvage treatment.

\section{Discussion}

This retrospective analysis confirms the previously reported prolonged survival of patients receiving ipilimumab. Brain metastases in patients with metastatic melanoma have commonly been regarded as conferring a worse prognosis in the context of an already grave disease. ${ }^{4}$ The anticipated control of brain metastases with available therapies has been low, and therefore their presence has often served as an exclusion criterion for patients undergoing treatment trials.

In this study, we reported an increase in survival due to ipilimumab use even in patients with brain metastases. In a cohort of 77 patients with melanoma brain metastases, median survival was increased from 4.9 to 21.3 months for individuals receiving ipilimumab. This finding supports the data obtained by Margolin et al. ${ }^{6}$ in a Phase II trial of ipilimumab for melanoma patients with brain metastases; that is, the drug had a similar level of activity in brain and nonbrain metastases. Responses in the brain lasted more than 12 months in some cases in that study.

All of the patients in the present study were enrolled in clinical trials prior to FDA approval of ipilimumab for advanced melanoma. Most were enrolled in compassionate use studies, which did not exclude individuals with brain metastases, treated or untreated. Prior trials have excluded patients with untreated brain metastases. Considering that previously untreated brain metastases have responded to ipilimumab therapy and the drug is now commercially available, we believe that SRS does not necessarily need to be performed for small, asymptomatic brain metastases before initiating ipilimumab.

Despite the finding that performance status, as represented by the DS-GPA score, was again identified as the most significant contributor to prolonged survival, ipilimumab remained a significant independent contributor to a higher survival probability (HR 0.48, 95\% CI $0.24-0.93, \mathrm{p}=0.03$ ) in a 24-month survival analysis. Although the 2-year survival probability in patients with metastatic melanoma has historically been in the range of only $10 \%-20 \% \%^{7,10}$ - which is supported by the rate $(19 \%)$ in our own group of patients who did not receive ipilimumab-the 2-year survival in patients treated with SRS and ipilimumab in our study was $47 \%$.

Further, the use of ipilimumab was associated with an increased survival probability at 2 years even when stratified by the DS-GPA score. Multiinstitutional data reported by Sperduto et al. ${ }^{13}$ showed that the overall median survival was 6.74 months (5.90-7.57 months), with 
J. P. S. Knisely et al.

TABLE 2: Baseline characteristics of melanoma brain metastasis in 77 patients*

\begin{tabular}{|c|c|c|c|c|}
\hline Characteristic & No. $(\%)$ & No Ipilimumab & Ipilimumab & $p$ Value \\
\hline $\operatorname{sex}$ & & & & $0.557 \dagger$ \\
\hline $\mathrm{F}$ & $28(36.4)$ & 17 & 11 & \\
\hline M & $49(63.6)$ & 33 & 16 & \\
\hline age in yrs & & & & $0.047 \ddagger$ \\
\hline median at diagnosis of metastasis & 61 & & & \\
\hline range & $24-89$ & & & \\
\hline mean $(95 \% \mathrm{Cl})$ & & $59.3(55.1-63.4)$ & $53.2(49.9-56.4)$ & \\
\hline $\mathrm{yr}$ of GKS & & & & $0.478 \dagger$ \\
\hline 2002 & $1(1.3)$ & 1 & 0 & \\
\hline 2005 & $4(5.2)$ & 4 & 0 & \\
\hline 2006 & $4(5.2)$ & 3 & 1 & \\
\hline 2007 & $15(19.5)$ & 9 & 6 & \\
\hline 2008 & $17(22.1)$ & 9 & 8 & \\
\hline 2009 & $20(26.0)$ & 15 & 5 & \\
\hline 2010 & $16(20.8)$ & 7 & 7 & \\
\hline location of primary melanoma & & & & $0.327 \dagger$ \\
\hline trunk & $36(46.7)$ & 22 & 14 & \\
\hline extremities & $19(24.7)$ & 12 & 7 & \\
\hline scalp, facial, auricular & $7(9.1)$ & 6 & 1 & \\
\hline distal acral & $7(9.1)$ & 3 & 4 & \\
\hline mucosal & $2(2.6)$ & 1 & 1 & \\
\hline unknown & $6(7.8)$ & 6 & 0 & \\
\hline chemotherapy & & & & $0.565 \dagger$ \\
\hline no & $29(37.7)$ & 20 & 9 & \\
\hline yes & $48(62.3)$ & 30 & 18 & \\
\hline targeted therapy & & & & $0.056 \dagger$ \\
\hline no & $63(81.8)$ & 44 & 19 & \\
\hline yes & $14(18.2)$ & 6 & 8 & \\
\hline \multicolumn{5}{|l|}{ received ipilimumab } \\
\hline yes & $27(35.1)$ & & & \\
\hline no & $50(64.9)$ & & & \\
\hline FU in mos & & & & $0.23 \ddagger$ \\
\hline median & 12.17 & 10.435 & 17.9 & \\
\hline mean & 18.69 & 17.15 & 21.55 & \\
\hline range & $1.97-72.83$ & $1.97-72.83$ & $6.6-50.93$ & \\
\hline WBRT & & & & $0.26 \dagger$ \\
\hline no & $52(67.53)$ & 36 & 16 & \\
\hline yes & $25(32.5)$ & 14 & 11 & \\
\hline alive at last FU & & & & $0.183 \dagger$ \\
\hline yes & $24(31.2)$ & 13 & 11 & \\
\hline no & $53(68.8)$ & 37 & 16 & \\
\hline ECOG PS & & & & $0.56 \dagger$ \\
\hline 0 & 45 & 27 & 18 & \\
\hline 1 & 25 & 18 & 7 & \\
\hline 2 & 7 & 5 & 2 & \\
\hline
\end{tabular}


Melanoma, stereotactic radiosurgery, and ipilimumab

TABLE 2: Baseline characteristics of melanoma brain metastasis in 77 patients* (continued)

\begin{tabular}{lcccc}
\hline Characteristic & No. (\%) & No Ipilimumab & Ipilimumab & $p$ Value \\
\hline DS-GPA & 16 & 13 & 3 & $0.21 \dagger$ \\
$0-1$ & 27 & 19 & 8 & \\
2 & 18 & 10 & 8 & \\
3 & 16 & 8 & & \\
& 4 & & & \\
$*$ FU = follow-up. & & &
\end{tabular}

corresponding median survivals for patients with DSGPA scores of $0-1,2,3$, and 4 of 3.38 months (2.73-4.27 months), 4.70 (4.17-5.42), 8.77 (6.83-10.77), and 13.23 (9.40-15.64). In our own cohort, among patients who did not take ipilimumab, those with a DS-GPA score of 0-2 had a median survival of 3.03 months, whereas those with a DS-GPA score of 3-4 had a median survival of 14.67 months. Both of these rates are comparable to those reported by Sperduto et al. ${ }^{13}$ In those who received ipilimumab in our study, however, the poor-prognosis patients with DS-GPA scores of 0-2 had a median survival of 15.67 months, and better-prognosis patients with DSGPA scores of 3-4 had a median survival of 25.2 months. These data suggest a substantial and unprecedented impact of ipilimumab on survival in patients with melanoma brain metastases - an observation that must be confirmed by aggregation of a larger patient cohort.

Ipilimumab is an active drug that has the potential for significant acute and delayed immune-mediated adverse events. In the study reported by Hodi et al., ${ }^{5}$ the frequency of Grade 3 or 4 immune-related adverse events was $10 \%-$ $15 \%$ in the ipilimumab groups and most often affected the skin and gastrointestinal tract, although endocrine and other adverse events were also seen. The most common reason for discontinuing therapy was disease progression, but over $60 \%$ of patients were able to take all 4 doses of ipilimumab. Fourteen deaths were related to the study drugs $(2.1 \%)$, and 7 were associated with immunerelated adverse events. The FDA's approval of ipilimumab was predicated on the development of a Risk Evaluation and Mitigation Strategy to accompany the drug to inform prescribers of the potential adverse effects. The strategies relate primarily to autoimmune colitis, which can be fatal. The present report specifically relates to our experience in patients with brain metastases.

Because the release of immunogenic molecules from radiosurgically treated tissues can be a protracted process, it is unclear at what point after SRS patients may be most at risk of having this marked inflammatory reaction. While some patients had focal therapy for their brain metastases prior to receiving ipilimumab, another subset demonstrated new brain metastases requiring SRS after ipilimumab treatment, and given the prolonged CTLA-4 blockade seen after ipilimumab administration, the possibility of immune-related CNS complications were an ongoing concern.

Specifically, we observed cases of necrosis causing symptomatic cerebral edema that required ongoing management with steroids. We have been reluctant to use protracted courses of dexamethasone, as this might adversely affect the efficacy of systemic CTLA-4 inhibition. Therefore, 3 patients required surgical evacuation of necrotic lesions because they could not be weaned from steroid therapy. Given the possibly extended survival that many patients responding to ipilimumab might enjoy, the reluctance to use steroid therapy in this group, and the understanding that the rate of symptomatic necrosis following radiosurgery increases with increased survival, the rate of surgical intervention in this group may be higher than in others receiving different systemic therapy. Further studies are needed to evaluate the use of bevacizumab in this setting and determine the frequency of severe CNS complications in patients undergoing SRS after receiving ipilimumab.

In this study we report on the natural history of CNS disease in patients receiving ipilimumab and treated with SRS alone without WBRT at time of initial diagnosis. Ipilimumab alone has been shown to shrink previously untreated brain metastases in only approximately $10 \%$ of patients. ${ }^{6}$ For patients in whom melanoma brain metastases develop during ipilimumab therapy, additional treatment of the metastases is often required. Most patients

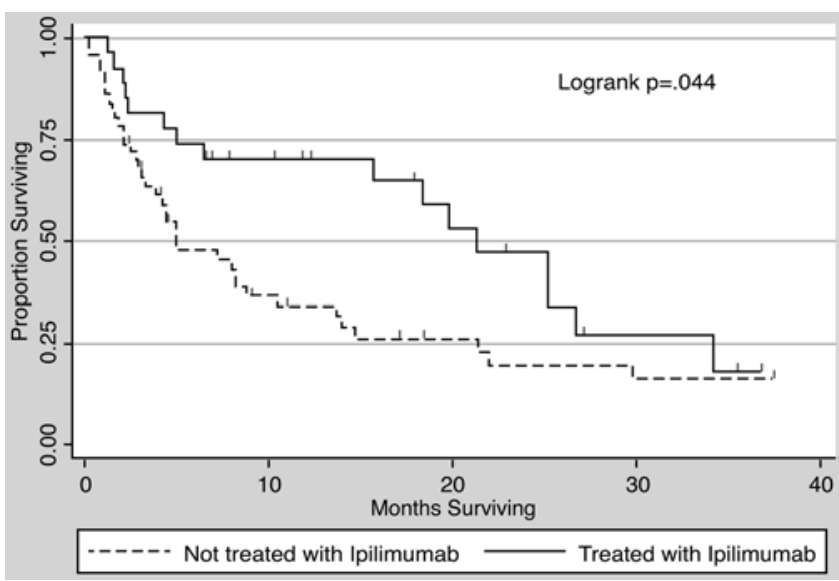

Fig. 1. Kaplan-Meier survival curve showing a median survival of 4.9 months (95\% Cl 3.3-10.4 months) for patients not receiving ipilimumab and 21.3 months $(95 \% \mathrm{Cl} 6.43-26.7$ months) in those receiving the drug. Two-year survival was $47.2 \%(95 \% \mathrm{Cl} 24.8 \%-66.8 \%)$ in the ipilimumab group compared with $19.7 \%(95 \% \mathrm{Cl} 9.0 \%-33.5 \%)$ in the nonipilimumab group. 
TABLE 3: Univariate and multivariate Cox proportional hazards regression, overall and with a 2-year survival cutoff

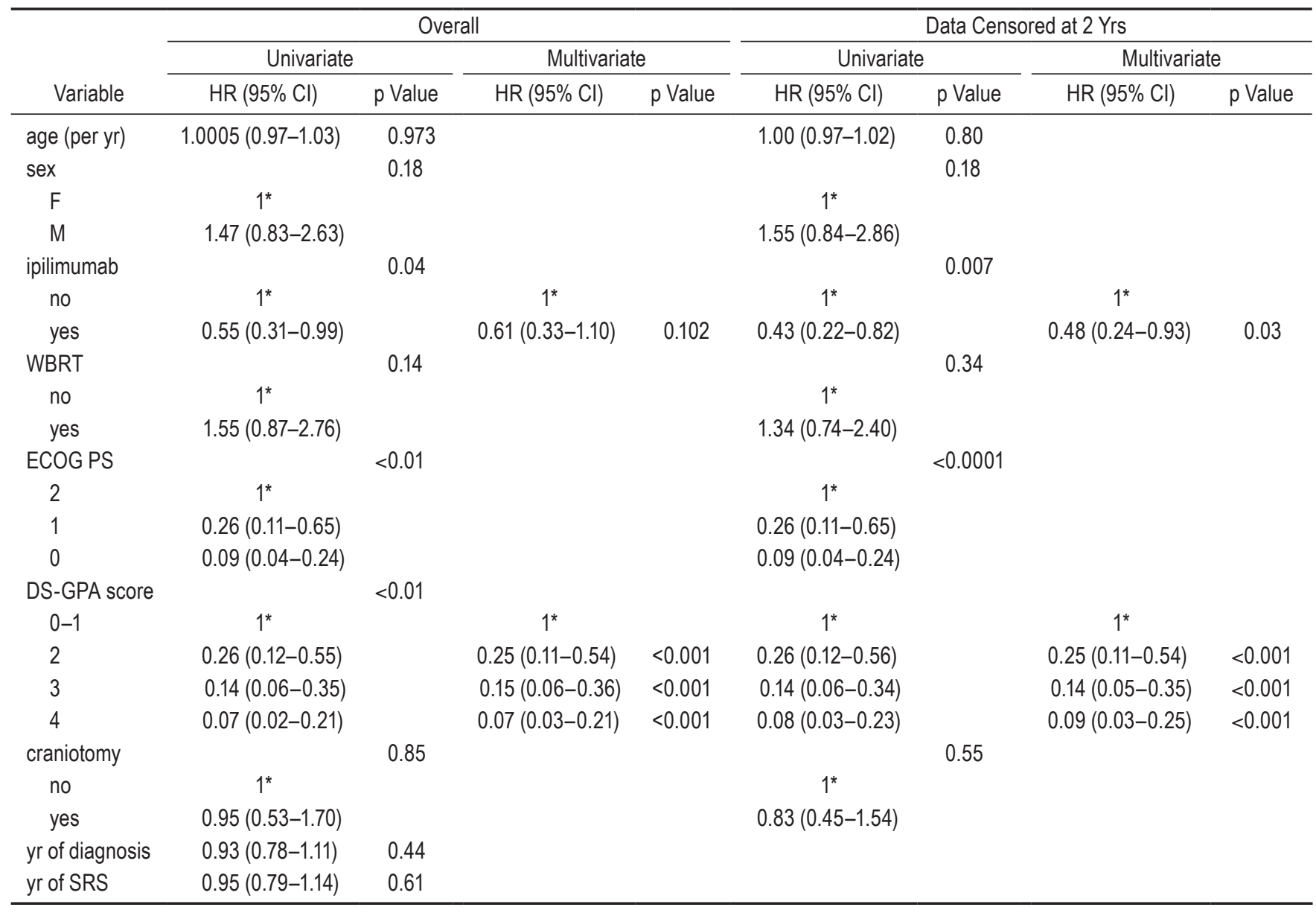

* Reference value.

with melanoma can avoid the recognized neurocognitive morbidity associated with WBRT through an approach that defers WBRT in preference for the use of SRS. Twothirds $(67.5 \%)$ of our patients never underwent WBRT, and $37 \%$ of those treated with ipilimumab never needed any CNS salvage therapy. The potential value of WBRT as part of salvage therapy cannot be addressed by this retrospective study since no data were specifically collected on the reasons for proceeding with WBRT. Several studies have provided Class I data documenting that the addition of WBRT to SRS in the initial management of

TABLE 4: Survival statistics for patients with better- and worse-prognosis melanoma brain metastasis

\begin{tabular}{lccc}
\hline & \multicolumn{3}{c}{ Median Survival (mos) } \\
\cline { 2 - 4 } \multicolumn{1}{c}{ Parameter } & DS-GPA Score & DS-GPA Score \\
& Overall & $0-2$ & $3-4$ \\
\hline DS-GPA survival statistics $^{*}$ & 6.7 & $2.73-5.42$ & $6.83-15.64$ \\
Yale SRS w/o ipilimumab & 4.9 & 3.03 & 14.67 \\
Yale SRS w/ ipilimumab & 21.3 & 15.67 & 25.2 \\
\hline
\end{tabular}

* As reported by Sperduto et al., 2010. patients with oligometastatic (fewer than 5) brain metastases confers no survival advantage and is associated with a neurocognitive impairment, although it does reduce the risk of intracranial recurrence. ${ }^{1,2}$ Despite the known higher rates of salvage therapy in patients treated with SRS alone, we were still able to report markedly improved survival in the present study, suggesting that the continuity and success of systemic control rather than the mode of CNS treatment is the important factor in determining overall survival.

We recognize that this study is small and that with the evolution of treatment paradigm shifts over the past decade our results may indeed be atypical. However, recruitment into the study was prospective and sequential, and the difference in survival at 2 years for those receiving ipilimumab was statistically dramatically better. A larger randomized study would help to validate or refute our findings.

\section{Conclusions}

This retrospective analysis showed that the survival of patients with metastatic melanoma treated with ipilimumab and supported preferentially via SRS control of brain metastases can far exceed the expected 4-6 months. 
Using ipilimumab in this treatment paradigm was associated with survival prolongation from 4.9 to 21.3 months and a population survival rate increase from $19.7 \%$ to $47.2 \%$ at 2 years. No statistical difference in survival was detected between patients in whom brain metastases developed before or after the initiation of ipilimumab. The association between the use of ipilimumab and prolonged survival remains significant even after adjustment for performance status. If SRS is used preferentially to provide primary control of brain metastases, our data suggest that patients will need on average 2 sessions of SRS and that about $20 \%$ of patients may ultimately require salvage WBRT. Complications may be observed within the CNS as well as peripherally as a result of ipilimumab's effects on the immune system. Further prospective randomized studies are warranted for determining the efficacy and optimal sequencing of ipilimumab and specific brain metastasis therapies in melanoma patients with brain metastases.

\section{Disclosure}

Dr. Sznol is an advisor to Bristol, Myers, Squibb.

This work was made possible by Clinical and Translational Science Awards Grant No. UL1 RR024139 from the National Center for Research Resources (NCRR), a component of the NIH, and NIH Roadmap for Medical Research. Its contents are solely the responsibility of the authors and do not necessarily represent the official view of the NCRR or the NIH. This work was also supported in part by the Milstein-Meyer Fund for Melanoma Research at Yale (M.S. and H.M.K.) and the Yale University Section of Medical Oncology Pilot Funding Program (H.M.K.).

Author contributions to the study and manuscript preparation include the following. Conception and design: Knisely, Chiang. Acquisition of data: Knisely, Flanigan, Sznol, Chiang. Analysis and interpretation of data: Knisely, Kluger, Chiang. Drafting the article: Knisely, Kluger, Chiang. Critically revising the article: all authors. Reviewed submitted version of manuscript: all authors. Approved the final version of the manuscript on behalf of all authors: Knisely. Statistical analysis: Yu. Administrative/technical/material support: Chiang. Study supervision: Knisely.

\section{Acknowledgment}

The authors acknowledge Judith Hess for meticulous attention to the Yale Gamma Knife database and the requirements of the Yale Human Investigations Committee.

\section{References}

1. Aoyama H, Tago M, Kato N, Toyoda T, Kenjyo M, Hirota S, et al: Neurocognitive function of patients with brain metastasis who received either whole brain radiotherapy plus stereotactic radiosurgery or radiosurgery alone. Int J Radiat Oncol Biol Phys 68:1388-1395, 2007

2. Chang EL, Wefel JS, Hess KR, Allen PK, Lang FF, Kornguth DG, et al: Neurocognition in patients with brain metastases treated with radiosurgery or radiosurgery plus whole-brain irradiation: a randomised controlled trial. Lancet Oncol 10: 1037-1044, 2009
3. Fertil B, Malaise EP: Intrinsic radiosensitivity of human cell lines is correlated with radioresponsiveness of human tumors: analysis of 101 published survival curves. Int J Radiat Oncol Biol Phys 11:1699-1707, 1985

4. Flanigan JC, Jilaveanu LB, Faries M, Sznol M, Ariyan S, Yu $\mathrm{JB}$, et al: Melanoma brain metastases: is it time to reassess the bias? Curr Probl Cancer 35:200-210, 2011

5. Hodi FS, O’Day SJ, McDermott DF, Weber RW, Sosman JA, Haanen JB, et al: Improved survival with ipilimumab in patients with metastatic melanoma. N Engl J Med 363:711-723, 2010

6. Margolin K, Ernstoff MS, Hamid O, Lawrence D, McDermott D, Puzanov I, et al: Ipilimumab in patients with melanoma and brain metastases: an open-label, phase 2 trial. Lancet Oncol 13:459-465, 2012

7. Middleton MR, Grob JJ, Aaronson N, Fierlbeck G, Tilgen W, Seiter S, et al: Randomized phase III study of temozolomide versus dacarbazine in the treatment of patients with advanced metastatic malignant melanoma. J Clin Oncol 18:158-166, 2000 (Erratum in J Clin Oncol 18:2351, 2000)

8. Retsas S, Gershuny AR: Central nervous system involvement in malignant melanoma. Cancer 61:1926-1934, 1988

9. Robert C, Thomas L, Bondarenko I, O’Day S, Weber J, Garbe $\mathrm{C}$, et al: Ipilimumab plus dacarbazine for previously untreated metastatic melanoma. N Engl J Med 364:2517-2526, 2011

10. Rusthoven JJ, Quirt IC, Iscoe NA, McCulloch PB, James KW, Lohmann RC, et al: Randomized, double-blind, placebocontrolled trial comparing the response rates of carmustine, dacarbazine, and cisplatin with and without tamoxifen in patients with metastatic melanoma. J Clin Oncol 14:20832090, 1996

11. Sampson JH, Carter JH Jr, Friedman AH, Seigler HF: Demographics, prognosis, and therapy in 702 patients with brain metastases from malignant melanoma. J Neurosurg 88:1120, 1998

12. Skibber JM, Soong SJ, Austin L, Balch CM, Sawaya RE: Cranial irradiation after surgical excision of brain metastases in melanoma patients. Ann Surg Oncol 3:118-123, 1996

13. Sperduto PW, Chao ST, Sneed PK, Luo X, Suh J, Roberge $\mathrm{D}$, et al: Diagnosis-specific prognostic factors, indexes, and treatment outcomes for patients with newly diagnosed brain metastases: a multi-institutional analysis of 4,259 patients. Int J Radiat Oncol Biol Phys 77:655-661, 2010

14. Staudt M, Lasithiotakis K, Leiter U, Meier F, Eigentler T, Bamberg M, et al: Determinants of survival in patients with brain metastases from cutaneous melanoma. Br J Cancer 102:1213-1218, 2010

Manuscript submitted November 4, 2011.

Accepted May 5, 2012.

Please include this information when citing this paper: published online June 15, 2012; DOI: 10.3171/2012.5.JNS111929.

Portions of this work were presented in poster form at the International Stereotactic Radiosurgery Society Congress held in Paris, France, on May 8-12, 2011, and at the American Society for Radiology and Oncology Meeting held in Miami, Florida, on October 2-6, 2011, and orally at the Society for Neuro-Oncology Meeting held in Garden Grove, California, on November 18, 2011.

Address correspondence to: Jonathan Knisely, M.D., Department of Radiation Medicine, North Shore University Hospital, 300 Community Drive, Manhasset, New York 11030. email: jknisely@nshs. edu. 